\title{
DÜBLIN
}

Technological University Dublin ARROW@TU Dublin

2004-01-01

\section{Analysis of Data Sets Using Trio Sonification}

Charlie Cullen

Technological University Dublin, charlie.cullen@tudublin.ie

Eugene Coyle

Technological University Dublin, Eugene.Coyle@tudublin.ie

Follow this and additional works at: https://arrow.tudublin.ie/dmccon

Part of the Other Computer Engineering Commons, and the Other Music Commons

\section{Recommended Citation}

Cullen, C. \& Coyle, E. (2004) Analysis of Data Sets Using Trio Sonification. ISSC: Irish Signals and Systems Conference. 30th June - 2nd July.

This Conference Paper is brought to you for free and open access by the Digital Media Centre at ARROW@TU Dublin. It has been accepted for inclusion in Conference papers by an authorized administrator of ARROW@TU Dublin. For more information, please contact arrow.admin@tudublin.ie, aisling.coyne@tudublin.ie, gerard.connolly@tudublin.ie.

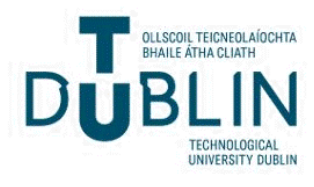




\title{
Analysis Of Data Sets Using Trio Sonification
}

\author{
Charlie Cullen $^{\phi}$ and Eugene Coyle* \\ ${ }^{\phi}$ Department of Music, \\ Dublin Institute of Technology, Dublin \\ IRELAND \\ * Department of Electronic Engineering, \\ Dublin Institute of Technology, Dublin \\ IRELAND \\ E-mail: ${ }^{\phi}$ charlie.cullen@dit.ie \\ E-mail:*eugene.coyle@dit.ie
}

\begin{abstract}
Recent advances in technology have suggested that sound and audio play a far greater part in our daily working lives than ever before. Mobile phone ring tones are now based upon polyphonic music sequences that allow relatively complex audio to be generated from a handset by way of conveying information (i.e. a call or message is incoming). This real world example of sonification suggests that far more could be made of sonification techniques for analysis- particularly in the business environment. One advantage of sonification is its relatively hands free nature in that once a sequence is being played it does not necessarily require further input from the user and so the potential exists for applications that could deliver information while other tasks are being performed in tandem.
\end{abstract}

For the definition of the basic principles of Trio sonification an application is being developed that will read in data sets of certain formats (.csv or .xml) and allow the various elements to be sonified for analysis. Existing work has suggested that many data elements can be conveyed within a single sonification and this would lend itself to analysis that seeks to take a broader assessment of a complete set of data.

Although the application is still in the development stage the techniques it will employ require consideration in their own rightnotably the use of rhythmic parsing to allow the conveyance of far greater levels of data using sound. It has been found that orchestration in general is of prime importance in effective and transparent data sonification and to this end the instrumentation and rhythmic arrangement of such data for analysis is effectively as much a technique in its own right as the sonification itself.

\section{ORCHESTRATED DATA ANALYSIS USING SONIFICATION}

The use of sonification as a means of data analysis is an ever-growing field of endeavour that will ideally come to be viewed in the same manner as visual graphs and charts as a means of representing data. Many different types of data have previously been sonified with a view to both composition and analysis, and although interesting and innovative the results can often suffer due to a lack of cohesion or transparency.
By applying sonification techniques to typical business data that has traditionally been the preserve of the spreadsheet or small database a different approach to data analysis can be utilised and hence evaluated with a view to its eventual integration in the workplace.

The key to the sonification of data is its orchestration [1] -that is the way the different elements of data are arranged relative to each other in terms of their instrumentation, melody and harmony and their rhythm. If a data element is represented by a melody then that melody must be harmonically and rhythmically distinct enough from the rest of the 
music that it can be detected by the listener among all the other data in the sonification. Conversely, the segregation of a data element to the extent of colouring the data unnecessarily (e.g. a strong melodic hook representing shoe size when analysing employee earnings will serve to shift the focus of the sonification in the wrong direction) is also a potential pitfall that has to be assiduously avoided.

In this respect, orchestration can be regarded in much the same manner as displaying different data elements on a graph or bar chart in that the use of resolution and colour can have a dramatic effect on the perception and hence the analysis of the data. This balance is of great importance in traditional visual representation techniques and hence must be afforded a similar effort in data sonification.

\subsection{Orchestration within sonification}

A musical orchestration can be described as a piece of music that has been balanced for performance by a particular set of voices or instruments [2]. The basic premise of orchestration as it is applied to modern music is the organisation of different voices or instruments playing different musical parts so that each part can be heard individually and also within the overall context of the piece as a whole.

Even at a basic level the use of orchestration allows groups of instruments to interact in a piece and still retain their individual parts within the whole work.

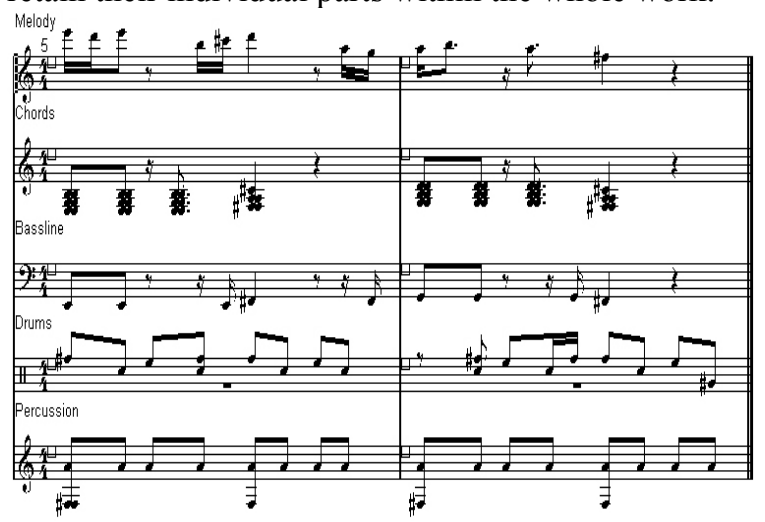

Figure 1: An example of basic orchestration in 5 parts

From the above score it can be seen that at least five different elements of data are being conveyed within a single musical passage, elements that themselves could be used to represent many different data values within a defined set.

The use of musical orchestration for the conveyance of greater amounts of information within even the most basic of music frameworks (e.g. bass, chords and melody) is potentially of great use within sonification, especially when used in conjunction with rhythmic patterns as a means of segmenting data for representation.
When arranging data in a sonification it is considered prudent to allocate data with greater consonance to a bass or percussion part, wherein small changes in data will be better highlighted, rather than trying to accommodate larger data sets of greater disparity within a more limited musical range. Conversely, data elements with greater variation are more suited to a chord interval or melody representation that would have sufficient scope to accommodate a wider range of data.

For example, a relatively static element defined in small groupings such as salary bracket of e5000 intervals would perhaps lend itself more to a bassline representation which, although changing during the piece would not be expected to change to such a degree that more than 24 different values would be required. As this data range could be well represented within 2 octaves it follows that an instrument such as the bass would be ideally suited to its rendering. However, if the salary were defined in smaller increments of e 1000 or less then a bassline representation would be inadequate for such a potential disparity of data. In this case a chord interval representation could be considered as a far more adaptable and robust solution that would accommodate the data set better than a bassline representation.

\subsection{Instrumentation within sonification}

Instrumentation can be defined as 'the art of combining the sounds of a complex of instruments to form a satisfactory blend and balance' [3] and as such is a technique that has much potential when applied in data sonification. The simple arrangement of different elements of the sonified data in terms of their sound and timbre allows a far more cohesive and robust template for sonification to be produced. By defining different data elements as specific instruments relative to the data they convey a far more transparent representation of the data can be achieved.

The choice of instruments used in a sonification can have great effect on its subsequent perception and analysis (much like different colours on a graph) and this choice is further curtailed by the general midi standard used in these sonifications [4]. The main aim of sonification is to deliver as much data within a single pass as possible without loss of clarity and so the choice of instrument is arguably as important as what it will play in the final sonification.

In a simple trio arrangement using the general midi standard for output, a bass instrument can easily be combined with a piano and a solo instrument such as a flute or guitar to produce simple and straightforward sonifications for data sets of three elements. Although this arrangement can be extended to include more data a simple trio serves as a good basis for analysing data without necessarily 
being a trained musician and should therefore allow the user to become more comfortable with the principles of sonification in as short a time as possible.

\section{TRIO SONIFICATION OF A BASIC EMPLOYEE DATABASE}

Many databases relate to the basics of employee wage structures and their corresponding data such as age, position, gender, attendance and so forth. It was felt that such a basic structure would be a good place to start when seeking to develop a more comprehensive framework for orchestration within data sonification, and hopefully any analysis could be performed in as straightforward a manner as possible. A basic database was set-up containing a number of fictional employees in a company. Within this framework various simple elements were defined for possible analysis [see Appendix].

Previous work on the use of rhythmic intervals as a delimiter for sonification of data suggested that any definition of the data in basic rhythmic terms should reflect elements of the data both common and important to all of the data in the set- effectively requiring a primary key [5] within the database structure. Rhythmic structure is potentially more important than melodic orchestration (particularly in large data sets or data which falls within a very narrow range of values) and so even at this stage it is important to define not only why each data value occurs but when.

In the database example, the definition of employee number was chosen as the primary key within the database, and its numerical and sequential nature make it ideal for defining each entry in the database. It must be noted that the allocation of primary and secondary keys in database schema design is not always based upon numerical values (e.g. alphabetical listings) but even within these structures it should be a relatively straightforward process to assign some form of numerical increment to each instance within the database and thus apply the principle of basic rhythmic delimiting.

\subsection{Bassline Definition}

With the allocation of a primary key defined as the basic delimiter for the whole sonification, other data elements could now be considered for analysis. The first element to be considered in a trio sonification should ideally be the bassline that will underpin all other elements overlaid upon it. In considering the element best suited to bassline representation the most important factor should be the consonance of data involved.

In the example database, employee salary is defined within e5000 levels (rather than as specific values) and so would be a suitable candidate for sonification.
Although other elements within the database could also be considered suitable due to the small data set defined here the final sonification template would not then be considered scalable in that salary brackets are unlikely to change dramatically in a short period of time- as opposed to an element like position or supervisor which could theoretically be altered on a far more regular basis.

In all of the following examples willfully simple and straightforward musical definitions have been used to keep the analysis process as clear as possible. In the definition of the bassline scale a simple $\mathrm{C}$ major scale has been chosen as the most easily understood scale within western music.

\begin{tabular}{|l|l|}
\hline $\begin{array}{l}\text { Salary Bracket } \\
(\mathrm{e} 5000 \text { levels })\end{array}$ & Bassline (Bass) \\
\hline $10,000-14,999$ & $\mathrm{C}$ \\
\hline $15,000-19,999$ & $\mathrm{D}$ \\
\hline $20,000-24,999$ & $\mathrm{E}$ \\
\hline $25,000-29,999$ & $\mathrm{~F}$ \\
\hline $30,000-34,999$ & $\mathrm{G}$ \\
\hline
\end{tabular}

Table 1: Salary level to bass note mapping

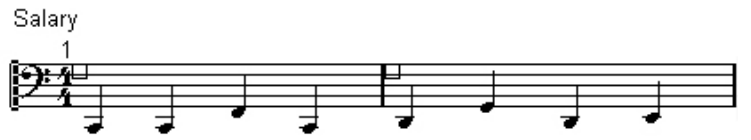

Figure 2: Bassline sonification of employee salary

\subsection{Chord Interval definition}

Following the same basic principles, the definition of chord intervals within a sonification would ideally be suited to elements within the data set that cover the greatest range of values. In this case, an element that contains relatively disparate data over a wider range could be better represented by some of the many combinations of chord intervals that a typical Pythagorean scale [6] provides us with.

From the above example, if the job title or position of an employee was considered for sonification alongside that employees salary then the use of chord intervals played on a piano for such a potentially diverse quantity could be of great use. The choice of piano for the chord intervals in the sonification follows from using acoustic bass within a basic trio format and is intended to allow for a degree of separation to be achieved between the two musical parts representing salary and position for each employee.

The chord intervals themselves are worthy of separate consideration for their melodic and harmonic content (as indeed is the bass scale) but for demonstration purposes simple major intervals are more than sufficient. 


\begin{tabular}{|l|l|}
\hline Position & Interval (piano) \\
\hline Shop Floor (SF) & (C\&E) \\
\hline StockControl(SC) & (D\&G) \\
\hline Purchasing (P) & (C\&Coct) \\
\hline Development (D) & (E\&A) \\
\hline Management (M) & (F\&B) \\
\hline
\end{tabular}

Table 2: Position to chord interval mapping

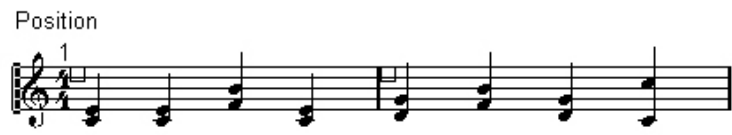

Figure 3: chord Interval sonification of employee position

In both of the above examples a wilfully straightforward approach has been taken to the melodic and harmonic structure of the data in order that the basic principles involved can be observed. It must be stated however that in much the same way as knowledge of chords and melody does not make a good composer neither does it serve as an ideal template for data sonification, with the above examples the technique itself is on display rather than the permutations it affords for analysis.

\subsection{Melody definition}

Many aspects of a data set are required to be highlighted as they occur within the data in order that they can be analysed within the overall set as a whole. The use of melodic signatures within a sonification is of great benefit when considering data of importance and its subsequent representation. Within any piece of music the single most prominent element is the melody (or melodies) and indeed when listeners are asked to describe a piece of music they will usually quote the melodic elements they have heard (n.b. this does not necessarily hold true for repeated in-depth analysis).

In order that melodic signatures be best utilised within a sonification it must first be decided what data they are intended to convey. With visual tools certain data elements are often considered of greater importance within the analysis and are therefore brought to greater prominence by the use of colour or scaling the graph or chart, a technique not dissimilar to the use of melodic signatures as a means of highlighting in sonification.

The instrument used for the melody within the sonification is also important in that it must stand out from the other instruments in terms of tone and texture, yet must also achieve a degree of acoustic consonance with the other instruments involved. To this end, a solo instrument like a flute is a useful choice in that it blends well with a bass and piano accompaniment while still retaining prominence within the ensemble as a whole. Many potential choices are available for all of the instruments used within a sonification and even the general midi standard allows for a reasonably disparate set of instrumental options when assigning data elements in sonification.

With reference to our previous examples, a basic melodic signature could be employed to highlight certain levels of seniority within the company structure such as management or skilled employees of certain types.

\begin{tabular}{|l|l|}
\hline Supervisor & Melody (flute) \\
\hline Supervisor 1 & $1^{\text {st }}, 3^{\text {rd }}, 5^{\text {th }}(C, E, G)$ \\
\hline Supervisor 2 & $5^{\text {th }}, 3^{\text {rd }}, 1^{\text {st }}(\mathrm{G}, \mathrm{E}, \mathrm{C})$ \\
\hline N/A & no melody \\
\hline
\end{tabular}

Table 3: Employee supervisor to melody mapping

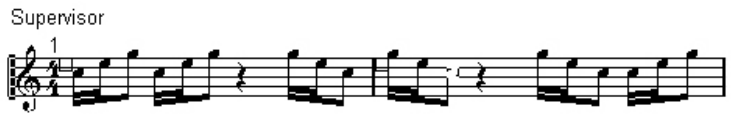

Figure 4: melody sonification of employee supervisor

The use of melody in this example would allow an employees salary and position in the company to be analysed together using a bassline and melody sonification and this could further be allied to the employees age as a chord interval.

Using just three basic instruments with very straightforward composition and orchestration a representation of an employees salary, age and position in the company can be sonified in a manner that allows for simple analysis.

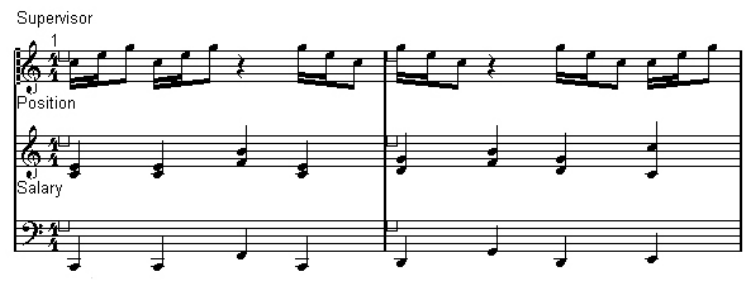

Figure 5: Basic trio sonification of employee data

This basic trio arrangement can be easily augmented with other instruments to allow for more data to be overlaid in the one single sonification that would ideally allow the user to pick out whatever information they require from the data set on the fly rather than by generating query upon query that will never show the overall relationships between larger groups of elements with any great degree of transparency.

\section{RHYTHMIC PARSING OF DATA FOR SONIFICATION}

As the data elements in the analysis begin to grow past a basic trio format so the brain becomes less able to distinguish between different instruments in the output sonification to the extent that the entire output becomes largely impenetrable in any way. In order to avoid this the rhythmic aspect of 
composition and orchestration is utilised to allow far greater amounts of information to be conveyed at the same time. In any performance of a musical piece the rhythm and time signature used are all-important in that they allow the listener the means to synchronise with the musical information they are receiving in order that the brain may process it accurately.

\subsection{Basic Rhythmic delimiters}

To convey multiple elements of a data set within a single sonification they must ideally be close enough together that the listener perceives them as related but not so close together that the become indistinguishable. Basic rhythmic patterns can be used as highly effective delimiters within a sonification to allow far greater amounts of data to be conveyed at once.

The first and most important aspect of rhythmic parsing in sonification is the time signature [7] to be used as it will dictate how many elements can be parsed together effectively before clarity begins to be lost. A 3/4 time signature has proven effective for other types of data sonified as part of this research [8] and other signatures may also prove equally useful depending on the data being analysed, but in keeping with the straightforward nature of the examples a 4/4 signature allows for a great degree of flexibility and familiarity to most listeners. Time Intervals

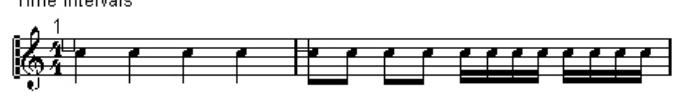

Figure 6: Basic rhythmic intervals

The use of $4 / 4$ gives subdivisions of 4 crotchets, 8 quavers and 16 semi-quavers within a single bar of time, and these subdivisions can be used as a means of parsing the different data elements requiring to be sonified. In the above example a basic trio of instruments was created that conveyed employee information regarding salary, age and position. It should be already noticeable that the basic rhythmic delimiter of a bar has been used as a means of incrementing each record in the data set- i.e. each employee has been allocated a bar of music within which all information about them required to be sonified must be conveyed.

\subsection{Rhythmic parsing of the employee database sonification}

It was found in initial testing that users kept up with initial sequences for a while but quickly fell behind or got confused to the point of being unable to differentiate between intervals they has already recognized earlier in the sequence. This problem was found to be largely associated with the lack of any perceivable rhythm or gap in the music that would allow the listener time to digest and process the information they had just heard, not unlike reading a book with no spaces or punctuation. The addition of basic rhythmic structures proved highly effective in allowing users to focus on smaller chunks of information in sequence and thus make a more informed judgment about their structure.

Subsequently, in the sonification of the employee database the addition of a basic crotchet rest delimiter in every bar (as in the DNASon software [8]) served to break the information into far more manageable segments, particularly in the bass line which would often be the first element to become indiscernible within even a basic test sequence (perhaps also due in part to the definition of pitches within a single octave major scale).

The orchestration was then segmented further by placing the chords and melody on different beats from the bass in order that different musical information within the sonification could be represented in as transparent a manner as possible. The use of the crotchet rest as a basic delimiter for all data in each bar gives way to smaller delimiters applied to specific elements of the data set as required. In this case, the melody line is offset by a semi-quaver from the beat of the bassline to give it greater definition.

The chord intervals were also offset by a quaver to give the user time to assess each event in its individual and collective entirety and also provided the fringe benefit of giving more movement to the rhythm of the sonification as a whole. With these basic delimiters in place, the final sonification of the employee database was rendered thus:

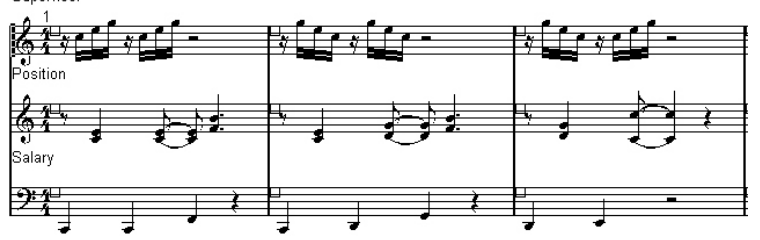

Figure 7: Final trio sonification of employee data

\section{FUTURE WORK}

Using the orchestration template for basic sonification, a simple database of employee related information has been sonified in a fairly straightforward and obvious manner. The emphasis is largely on the obvious, as the templates suggested by such basic orchestration techniques will hopefully lend themselves to sonifications that can be easily analysed and understood by any and all regardless of musical knowledge or experience. The principles defined here are intentionally simple in order that they may eventually be utilized within a non-musical analysis framework (i.e. by users who potentially have no musical education or training whatsoever).

It is perceived as vital to translate many of the accomplished methods used in sonification into a 
framework that can be manipulated and hence understood at a general level so that it becomes a more integrated part of data analysis in general. To this end, a new application is being developed in the spirit of the existing DNASon software that will ideally deal with business data of various formats. It is hoped that eventually an application can be developed that takes fairly basic data sets and sonifys them in a similarly obvious manner for the purposes of analysis and hence development.

It is eventually hoped to produce a basic application that will sonify data provided within a standard delimited database format such as XML (extensible markup language) or CSV (comma separated values). This application will ideally provide the basis for more investigation into the orchestration and rhythmic parsing of sonification data, as the initial work suggests that much could be obtained from such an endeavour.

In the final application the output sonification will be displayed as both a musical score (perhaps not a standard notation) alongside a graph of the results being analysed in order that both methods of data display can be used as required. This integrated display method is intended to give the user as much information about a data set as they require in a manner that will allow both in-depth and overview analysis of the same data in a single pass.

\section{REFERENCES}

[1] The Study of Orchestration, Samuel Adler, W.W Norton \& Company, ISBN 0-393-97572-X

[2] Orchestration, Walter Piston, W.W Norton \& Company, ISBN 0-393-09740-4

[3] The New Grove Dictionary of Music and Musicians $2^{\text {nd }}$ edition volume 12 , Macmillan Publishers ltd 2001, ISBN 0-333-60800-3

[4]The Midi Files, Rob Young, Prentice Hall, ISBN $0-13-262403-6$

[5] Database Systems $3^{\text {rd }}$ Edition (pg79), Thomas Connolly and Carolyn Begg, Addison Wesley, ISBN 0-201-70857-4

[6] Sound and Recording an Introduction $3^{\text {rd }}$ edition, Rumsey \& McCormack, Focal Press, ISBN 0-24051487-4

[7] The AB Guide to Music Theory Parts 1\&2, Eric Taylor, ABRSM Publishing, ISBN 1-85472-446-0

[8] Rhythmic Parsing of Sonified DNA and RNA Sequences, Charlie Cullen and Eugene Coyle, ISSC2003 Limerick.

\section{APPENDIX}

Basic Employee Database as used in test sonifications

$\begin{array}{llllll}\begin{array}{l}\text { Employee } \\ \text { No } \\ 1\end{array} & 1^{\text {st }} \text { Name } & 2^{\text {nd }} \text { Name } & \text { Position } & \text { Salary } & \text { Svisor } \\ 2 & \text { Joe } & \text { Soap } & \text { Shop Floor } & 12,000 & \text { J Doe } \\ 3 & \text { Mary } & \text { Soap } & \text { Shop Floor } & 14,000 & \text { J Doe } \\ 4 & \text { John } & \text { Doe } & \text { Management } & 28,000 & \\ & \text { Jane } & \text { Doe } & \text { Shop Floor } & 11,000 & \text { S } \\ 5 & \text { James } & \text { Watt } & \text { Stock } & 18,000 & \text { S } \\ 6 & \text { Susan } & \text { Watt } & \text { Control } & \text { Management } 33,000 & \text { Watt } \\ 7 & \text { Tony } & \text { Curtis } & \text { Stock } & 17,000 & \text { S } \\ & & & \text { Control } & & \text { Watt } \\ 8 & \text { Julia } & \text { Curtis } & \text { Purchasing } & 22,000 & \text { J Doe }\end{array}$

